\title{
Special Issue: Robot Motion and Control
}

\section{Krzysztof Kozłowski ${ }^{1}$}

Published online: 3 September 2018

(C) The Author(s) 2018

This special issue includes extended versions of selected papers presented during the 11th International Workshop on Robot Motion and Control, held in Wąsowo, Poland, July 3-5, 2017, an international event cosponsored by IEEE Robotics and Automation Society, Robotics and Automation Chapter, IEEE Polish Section, IEEE Control Systems Society, Polish Society for Measurements, Automatic Control and Robotics, Polish NMO of IFAC. The Workshop was organized under the auspices of the Institute of Automation and Robotics of the Poznań University of Technology, Poland.

43 original works have been selected for oral presentation of the 11th International Workshop on Robot Motion and Control. In average each paper has got three reviews and based on the reviewers' comments all accepted papers were corrected and finally appeared in the IEEE Explore [1].

During the Workshop five plenary presentations were given: Professor Witold Respondek Laboratoire de Mathématiques, INSA de Rouen, France, Kimon P. Valavanis, University of Denver, Denver, USA, Professor Luonis Adouane, Institute Pascal, UMR CNRS/UCA/SIGMA Polytech Clermont-Ferrand France, Professor Antŏnio Pedro Aguiar, University of Porto, Portugal, and Professor Yongchun Fang, Nankai University, Tianjin, China.

Following kind invitation and suggestion of Professor Kimon P. Valavanis the authors of 12 best papers presented during the Workshop were invited to submit an extended version to the special issue on Robot Motion and Control of the Journal of Intelligent and Robotic Systems. The Members of the International Scientific Committee selected the best presentations. The authors were asked to extend their papers presented during the Workshop with at least $60 \%$ of a new material.

Krzysztof Kozłowski

krzysztof.kozlowski@put.poznan.pl

1 Poznań University of Technology, Poznań, Poland
After a rigorous review process 11 papers were selected and accepted to be published in this special issue based on minimum of two specialized reviewers' opinion with the similarity score less then $40 \%$, actually this requirement was more strict and was approximately $30 \%$ for each individual paper. They are listed below:

1. K. Tchoń, W. Respondek, J. Ratajczak, Normal forms and configuration singularities of a space manipulator.

2. M. Galicki, Tracking of kinematically optimal trajectories by mobile manipulators.

3. I. Pająk, Real-time trajectory generation methods for cooperating mobile manipulators subject to state and control constraints.

4. M. Janiak, Ł. Chojnacki, Closing the loop-predicative Lifted Newton trajectory tracking algorithm.

5. W. Kowalczyk, Rapid navigation function control for two-wheeled mobile robots.

6. W. Domski, A. Mazur, M. Kaczmarek, Extended factitious force approach for control of mobile manipulator moving on unknown terrain.

7. Malik M.A. Al-Isawi, J. Sasiadek, Guidance and control of a robot capturing an uncooperative space target.

8. D. Belter, J. Wietrzykowski, P. Skrzypczyński, Employing natural terrain semantics in motion planning for a multi-legged robot.

9. V. Dutta, T. Zielińska, Predicting human actions taking into account object affordance.

10. V. Vonásek, A. Jurčik, K. Furmanová, B. Kozliková, Sampling-based motion planning for tracking evolution of dynamic tunnels in molecular dynamics simulations.

11. C. Zieliński, M. Figot, R. Hexel, Communication within Multi-FSM based robotic systems.

Paper no 1 presents the problem of normal forms and singularities for nonholonomic robotic systems represented by control-affine systems. The main idea of this paper is to rely the analysis of configuration singularities on normal forms 
of robotic system and this idea was applied to the dynamics of a space manipulator. Normal forms of this manipulator under the feedback equivalence were obtained and exploited in the analysis of its configuration singularities.

Paper no 2 is addressing the kinematically optimal control problem of the mobile manipulators. A computationally simple class of the Jacobian transpose control algorithms is proposed for the end-effector trajectory tracking. The performance of the proposed control strategies is illustrated through computer simulations for a mobile manipulator that attains trajectory tracking by the end-effector in a twodimensional task space and simultaneously minimizes some objective function.

In paper no 3 author solved the problem of real-time trajectory generation for two cooperating mobile robots moving the common rigid object. The holonomic constraints due to a closed kinematic chain along with collision avoidance conditions of such a system are considered. Two solutions of trajectory generation are proposed, namely firstly leader-follower approach is suggested and secondly the robotic system is treated as a whole mechanism. A computer simulation of two mobile manipulators consisting of nonholonomic platform $(2,0)$ class and planar 3DoF holonomic manipulator is presented.

Paper no 4 is concentrated on a predictive closed-loop trajectory tracking algorithm for nonlinear control systems that combines the Model Predictive Control (MPC) with the task priority Lifted Newton method. Performance of the closed-loop predictive Lifted Newton trajectory tracking algorithm is illustrated by series of computer simulations for the kinematic car like platform.

In paper no 5 a new gradient algorithm of the navigation function is introduced for a kinematic controller of a differentially driven mobile robot and velocity is computed as a function of position and orientation errors. Analysis of the convergence shows how the location of the eigenvalues of linearized system affects time of goal achievement and transient states. Extended experiments illustrate the proposed approach, saddle point detection and avoidance methodology.

In paper no 6 authors present a new control algorithm for mobile manipulators with skid-steering platform, moving on unknown terrain. Authors proposed to use the concept of factitious force and discussed a new adaptive and robust control law that guarantees asymptotically fast convergence of tracking errors to zero. Conducted simulations illustrate the proposed control law showing that it works properly even in terrain with parameters that are unknown and piecewise constant.

Paper no 7 presents a new method to guide and control a space robot for capturing an uncooperative target. The dynamic model of a target is unknown and estimated using vision system. A detailed description of the methodology consisting of three steps is described in this paper. A new trajectory method for planning the end-effector velocities of the space robot arm was implemented based on the measurement information from the vision system and Adaptive Unscented Kalman Filter. Simulation experiments are presented and discussed.

In paper no 8 authors discuss motion planning for a sixlegged walking robot in rough terrain, considering both the geometry of the terrain and its semantic labelling. Popular classification algorithms were tested such as Support Vector Machine and Random Trees. Reported experimental results show the ability of the new approach to avoid areas that are considered risky for legged locomotion.

Paper no 9 is presenting the probabilistic method for action prediction in human-object interactions. The key idea of the proposed approach is the description of the socalled object affordance, the concept that allows delivering trajectory visualization possible future actions. Extensive experiments are reported showing the effectiveness of the proposed method in action prediction. The dataset recorded contains several human activities composed out of different actions.

Paper no 10 presents dynamic behaviour of proteins that depends on the presence of internal void space known as tunnels or cavities. Authors propose a method that enables to trace tunnels. The Rapidly Exploring Random Tree algorithm is used to explore the void space in each frame of the protein dynamics. The results of the proposed method are demonstrated on an exemplary dataset obtained from the domain experts and the results are compared with the classic aggregation-based tunnel detection using state-of-the-art CAVER 3.0 tool.

In paper no 11 authors present a robotic system design methodology based on the concept of an embodied agent decomposed into communicating systems. This methodology is exemplified with a rudimentary table tennis ballcollecting robot. The presented methodology and the implementation tools are suitable and beneficial for application to the design of other robotic systems.

I strongly believe that these papers due to new theoretical and experimental results in robot control would be of interest to the Readers of the Journal of Intelligent and Robotic Systems.

I would like to express my sincere appreciation to the authors of this Special Issue for their contribution and the cooperation during the review process. Many thanks are forwarded to all reviewers for their insightful and thorough reviews during the review process of this Special Issue. Their valuable comments and suggestions 
contributed significantly to the improvement of the papers quality.

Special thanks are forwarded to Professor Kimon $\mathrm{P}$. Valavanis, Editor-in-Chief of the Journal of Intelligent and Robotic Systems for joining and delivering plenary presentation at the IEEE International Workshop on Robot Motion and Control in 2017, initiating this issue and support at the each stage of the review process.

Open Access This article is distributed under the terms of the Creative Commons Attribution 4.0 International License (http:// creativecommons.org/licenses/by/4.0/), which permits unrestricted use, distribution, and reproduction in any medium, provided you give appropriate credit to the original author(s) and the source, provide a link to the Creative Commons license, and indicate if changes were made.

Publisher's Note Springer Nature remains neutral with regard to jurisdictional claims in published maps and institutional affiliations.

Krzysztof Kozłowski received the M.Sc. degree in electrical engineering from Poznan University of Technology (PUT), Poland, and the Ph.D. degree in control engineering from PUT in 1979, where he currently holds full professor position in robotics and automation. In 1993, he was a Fulbright scholar with Jet Propulsion Laboratory, Pasadena, USA. He founded and serves as a chairman of a new Institute of Automation and Control established on May 1, 2017 at Poznan University of Technology.
He teaches and conducts research in the area of modeling and control of industrial and mobile robots. His research interests include multi-agent systems, identification and various robotics applications. His research publications include more than 140 conference papers and more than 65 papers published in national and international journals. He is an author of the book titled Modelling and Identification in Robotics (Springer-Verlag, 1998).

He was an Associate Editor for the IEEE Transactions on Control Systems Technology (1999-2008), for the IEEE Robotics and Automation Magazine (1998-2002), for the Journal of Intelligent and Robotic Systems (2005-2010) and for the International Journal of Applied Mathematics and Computer Science, (1999-2017), IEEE Conference Editorial Board, Conference on Decision and Control (CDC) and American Control Conference (ACC) since 1999 till now. He was the member of the Administrative Committee, IEEE Robotics and Automation Society 2000-2002, 2004-2005 and the member of the Board of Directors, IEEE Control Systems Society, 2003-2004. He serves as the chair of the IEEE Robotics and Automation Chapter, Polish Section, 2000-2008 and 2014-2019.

He conducted 39 different research scientific projects as supervisor (26) and as principal investigator (13) granted by national research institutions and 4 international research agencies.

\section{References}

1. RoMoCo. https://ieeexplore.ieee.org/xpl/mostRecentIssue.jsp?punum ber=7999195 (2017) 